\title{
ENFERMAGEM E VISIBILIDADE NA PANDEMIA DA COVID-19: MONITORAMENTO DE MÍDIA SOCIAL
}

\section{NURSING AND VISIBILITY IN COVID-19 PANDEMIC: SOCIAL MEDIA MONITORING}

\section{ENFERMERÍA Y VISIBILIDAD EN LA PANDEMIA DEL COVID-19: MONITORIZACIÓN DE LOS MEDIOS SOCIALES}

\author{
Tiago Oliveira de Souza ${ }^{1}$ \\ Hércules Rigoni Bossato ${ }^{2}$ \\ Isabela Barboza da Silva Tavares Amaral ${ }^{3}$ \\ Fernanda Teles Morais do Nascimento ${ }^{4}$ \\ Ítalo Rodolfo Silva ${ }^{5}$ \\ Luana dos Santos Costa ${ }^{6}$
}

\begin{abstract}
Como citar este artigo: Souza TO, Bossato HR, Amaral IBST, Nascimento FTM, Silva IR, Costa LS. Enfermagem e visibilidade na pandemia da COVID-19: monitoramento de mídia social. Rev baiana enferm. 2021;35:e38740.

Objetivo: analisar as publicações de usuários do Twitter direcionadas à campanha de valorização da Enfermagem, impulsionadas pelos termos \#LuteComoUmaEnfermeira e \#EnfermagemEuValorizo. Método: pesquisa quantitativa, fundamentada nos métodos digitais. A busca foi realizada utilizando as hashtags \#LuteComoUmaEnfermeira e \#EnfermagemEuValorizo para extração de tweets. Foram selecionados para análise 2.613 tweets. Resultados: 22,5\% das publicações localizam-se na região Sudeste do país e 9,3\% na região Nordeste. Houve mais de 18 mil interações (curtidas e compartilhamentos), sendo que os autores das postagens atingem um público estimado de cerca 7 milhões de pessoas. O conteúdo das postagens versava principalmente sobre a regulamentação da jornada de trabalho, Equipamentos de Proteção Individual, combate ao COVID-19, defesa da força de trabalho majoritariamente feminina e luta por direitos. Conclusão: a realização da campanha virtual teve visibilidade importante no território nacional e apresentou grande visibilidade à profissão em número e alcance na Internet.
\end{abstract}

Descritores: Infecções por Coronavírus. Informática em Enfermagem. Profissionais de Enfermagem. Mídias Sociais. Mineração de Dados.

Objective: analyze the publications of Twitter users directed to the campaign of valuation of Nursing, driven by the terms \#LuteComoUmaEnfermeira and \#EnfermagemEuValorizo. Method: quantitative research, based on digital methods. The search was realized using the hashtags \#LuteComoUmaEnfermeira and \#EnfermagemEuValorizo for tweets extraction. 2,613 tweets were selected for analysis. Results: 22.5\% of publications are located in the Brazilian Southeast region and $9.3 \%$ in the Northeast region. There were more than 18,000 interactions (likes and shares),

Enfermeiro. Doutor em Saúde Pública. Professor de Enfermagem em Saúde Coletiva na Universidade Federal do Rio de Janeiro, Campus Macaé. Rio de Janeiro, Rio de Janeiro, Brasil. tiagotos@gmail.com. http://orcid.org/0000-0002-0926-2926.

Enfermeiro. Doutor em Enfermagem. Professor de Enfermagem em Saúde Coletiva na Universidade Federal do Rio de Janeiro, Campus Macaé. Macaé, Rio de Janeiro, Brasil. http://orcid.org/0000-0002-0443-8996.

3 Enfermeira. Mestre em Ciências do Cuidado em Saúde. Professora de Enfermagem em Saúde Coletiva da Universidade Federal do Rio de Janeiro, Campus Macaé. Macaé, Rio de Janeiro, Brasil. http://orcid.org/0000-0003-4043-6020.

Enfermeira. Doutora em Enfermagem e Biociências. Professora Adjunta de Enfermagem em Saúde Coletiva na Universidade Federal do Rio de Janeiro, Campus Macaé. Macaé, Rio de Janeiro, Brasil. http://orcid.org/0000-0002-0I20-5687.

5 Enfermeiro. Doutor em Enfermagem. Professor de Metodologia da Assistência e do Ensino de Enfermagem da Universidade Federal do Rio de Janeiro, Campus Macaé. Professor Permanente no Programa de Pós-Graduação em Enfermagem da Escola de Enfermagem Anna Nery. Macaé, Rio de Janeiro, Brasil. http://orcid. org/0000-0002-2882-1877.

6 Enfermeira. Mestre em Enfermagem. Especialista em Terapia Intensiva em Adulto. Rio de Janeiro, Rio de Janeiro, Brasil. http://orcid.org/0000-000 I -73 I 4-3676. 
and the authors of the posts reach an estimated audience of about 7 million people). The content of the posts mainly related to the regulation of working hours, Personal Protective Equipment, the fight against COVID-19, the defense of the majority female workforce and the fight for rights. Conclusion: the realization of the virtual campaign had important visibility in the national territory and presented great visibility to the profession in number and reach on the Internet.

Descriptors: Coronavirus Infections. Informatics in Nursing. Nursing Professionals. Social Media. Data Mining.

Objetivo: analizar las publicaciones de los usuarios de Twitter dirigidas a la campaña de valoración de la Enfermería, impulsada por los términos \#LuteComoUmaEnfermeira y \#EnfermagemEuValorizo. Método: investigación cuantitativa, basada en métodos digitales. La búsqueda se llevó a cabo utilizando los hashtags \#LuteComoUmaEnfermeira y \#EnfermagemEuValorizo para la extracción de tweets. Resultados: el 22,5\% de las publicaciones se localizan en la región Sudeste del país y el 9,3\% en la región Nordeste. Hubo más de 18.000 interacciones (curtidas y compartidas), y los autores de los mensajes llegaron a un público estimado de unos 7 millones de personas. El contenido de los puestos se refería principalmente a la regulación de los borarios de trabajo, el equipo de protección personal, la lucha contra el COVID-19, la defensa de la fuerza de trabajo mayoritariamente femenina y la lucha por los derechos. Conclusión: la realización de la campaña virtual tuvo una importante visibilidad en el territorio nacional y presentó una gran visibilidad a la profesión en número $y$ alcance en Internet.

Descriptores: Infecciones por Coronavirus. Informática Aplicada a la Enfermería. Enfermeras Practicantes. Medios de Comunicación Sociales. Minería de datos.

\section{Introdução}

Em dezembro de 2019, um novo Coronavírus, SARS-CoV-2, foi identificado na cidade de Wuhan, província de Hubei, China ${ }^{(1)}$. O vírus tem alta transmissibilidade e provoca uma síndrome respiratória aguda que se espalhou para vários países do mundo. A doença, COVID-19, dessa forma, deixa de ter um caráter local e passa rapidamente a se tornar uma pandemia ${ }^{(2)}$. Ela varia de casos leves a casos muito graves com insuficiência respiratória que acometem cerca de 5\% e 10\% dos casos, necessitando de imediata internação devido à gravidade do quadro clínico.

Isto posto, o Brasil adotou um plano de contingência e medidas de resposta relacionadas às ações de contenção e mitigação para diminuir o avanço da pandemia que variou, em dinâmica, de estado para estado da federação ${ }^{(3)}$.

Nesse contexto, os profissionais de Enfermagem assumem relevante papel no combate ao novo coronavírus e mediante o gerenciamento e práticas de cuidado às pessoas com COVID-19, estabelecem o planejamento e as intervenções na linha de frente do enfrentamento da pandemia. Sendo assim, a Enfermagem tem atuado no cuidado direto desde a atenção primária à saúde, com o acompanhamento dos casos em isolamento domiciliar até a atenção terciária, com os cuidados à pessoa internada em estado grave, utilizando de competências para atuação no cuidado crítico ao encontro das demandas clínicas provocadas pela doença em evidência.

Nessa conjuntura, há evidências das dificuldades da equipe de enfermagem relacionadas às frágeis condições de trabalho, sobrecarga física e emocional, remuneração precária e falta de capital humano e ferramentas, como, por exemplo, equipamentos de proteção individual (EPIs), tão necessários para o enfrentamento da pandemia de COVID-19 ${ }^{(4)}$.

Em ampla contextualização, cabe ressaltar que no mundo do trabalho há diversas categorias profissionais que vêm sofrendo a deterioração das condições trabalhistas e a perda de direitos devido ao ajuste fiscal e ao corte de investimentos pelo Estado, condicionados pela financeirização do sistema capitalista. Nesse contexto, observa-se que a Enfermagem brasileira vem sofrendo redução de recursos humanos, manutenção de jornada extensiva de trabalho, deterioração da qualidade de vida, perda de 
direitos, baixo salário e vínculos de trabalhos precários e frágeis ${ }^{(5)}$.

Assim, paralelo às exposições globais da macropolítica relacionadas à pandemia, é preciso destacar a importância das micropolíticas, contextos onde ocorrem os espaços de subjetividades humanas, mas que concretizam as realidades locais, alinhadas às tendências globais. Nesse sentido, em meio à necessidade de manutenção do isolamento social e adoção das medidas recomendadas pela Organização Mundial de Saúde (OMS), no Brasil, um grupo de profissionais de Enfermagem que protestavam de forma pacífica por medidas de melhores condições de trabalho para a categoria atuante na pandemia, foi surpreendido com ataques de grupos que supostamente contrariavam as orientações científicas preconizadas pela OMS de contenção da pandemia.

A manifestação e a luta dos enfermeiros, nesse cenário, configurava-se em reivindicações por mais recursos para a saúde e o luto pelas mortes causadas pela COVID-19. Ademais, cabe registrar que o Brasil lidera atualmente o recorde de profissionais de enfermagem mortos por COVID-19 ${ }^{(6)}$.

Nesse contexto, tanto do protagonismo de Enfermagem no enfrentamento da pandemia quanto da luta, em protesto, da categoria por valorização profissional e requisição de maiores investimentos do governo federal no Sistema Único de Saúde (SUS), é que entidades organizativas da profissão, como o Conselho Federal de Enfermagem (COFEN), têm realizado campanhas de promoção da valorização da categoria. Entre essas destaca-se o "Ato Virtual pela Enfermagem aos profissionais mortos na Pandemia", realizado no dia 12 de maio de 2020, Dia Internacional do Enfermeiro, e que mobilizou, via Internet, a publicação de mensagens, imagens, vídeos e informações, com a finalidade de dar visibilidade à valorização da Enfermagem.

Desse modo, foi objetivo desta pesquisa analisar as publicações de usuários do Twitter direcionadas à campanha de valorização da Enfermagem, impulsionadas pelos termos \#LuteComoUmaEnfermeira e \#EnfermagemEuValorizo.

\section{Método}

Trata-se de pesquisa quantitativa, fundamentada nos métodos digitais ${ }^{(7)}$ e realizada por meio de uso de ferramentas e técnicas de mineração de dados em mídia social virtual. A pesquisa tem por finalidade refletir, descrever e discutir aspectos relacionados à Enfermagem, no campo de conhecimento da Informática em Enfermagem. Esse campo da ciência da informação ocupa-se com a análise e a disseminação de dados mediante o uso de dispositivos, principalmente ligados à Internet ${ }^{(8)}$.

Atualmente o Twitter representa uma importante ferramenta para as pessoas comunicarem rapidamente as suas opiniões, veicular informações e manifestar posicionamentos acerca de algum tema. O Twitter é uma mídia social e um servidor no qual seus usuários publicam e compartilham conteúdos (textos curtos de até 280 caracteres - tweets), imagens e outras mídias específicas e permite a interação entre outros usuários que curtem, comentam e compartilham (retweet) as publicações ${ }^{(7,9)}$. Todos esses elementos interativos implicam o engajamento entre as pessoas nessas plataformas sociais. Ademais, uma vantagem de utilizar a ferramenta para pesquisas é o fato de sua Interface de Programação de Aplicativos (Application Programming Interface (API)) permitir a obtenção e a extração de grande quantidade de dados que podem ser analisadas e gerar informação de valor e de caráter interdisciplinar em ciência de dados.

Os dados foram obtidos de um sistema de coleta automático de tweets utilizando os softwares RStudio e Facepager, com base no script elaborado para a pesquisa e de pacote do $\mathrm{R}$ (rtweet ${ }^{(10)}$. A extração de dados deu-se por meio da API. Essa técnica envolve uma interconexão entre o RStudio e a API do Twitter, e essa conexão criada possibilita a coleta de dados. Esse processo de mineração, via API, demanda certo conhecimento de execução de comandos e programação ${ }^{(9)}$.

A busca foi realizada um dia após a campanha virtual (13 de maio de 2020) sobre a Enfermagem e os profissionais mortos na pandemia 
da COVID-19, utilizando para extração de tweets as hashtags \#LuteComoUmaEnfermeira e \#EnfermagemEuValorizo. Os dados foram encontrados com a utilização das hashtags entre o período de 5 e 13 de maio de 2020. A hashtag é uma palavra-chave antecedida pelo símbolo cardinal (\#), que permite que as publicações na Web possam ser mais facilmente encontradas. Termos, palavras-chaves ou tags frequentemente são utilizados para indexar uma discussão ou tópico no Twitter, Facebook, Google, Youtube e Instagram.

Desse modo, foi localizada uma amostra de 3.356 tweets originais de usuários da mídia social. Foram excluídos 743 tweets duplicados (do mesmo usuário/autor) e restaram 2.613 tweets selecionados para análise.

Os critérios de inclusão foram tweets que utilizaram as hashtags da campanha (\#LuteComoUmaEnfermeira ou \#EnfermagemEu-Valorizo) publicadas durante o período de análise do estudo. Os retweets (RT) foram excluídos e apenas foram contabilizados o número de RT (compartilhamentos) dos tweets/mensagens originais.

A fim de obtermos uma amostra de tweets pessoais, isto é, publicados diretamente por usuários de contas pessoais, coletou-se tweets publicados por softwares de clientes conhecidos: dispositivos Android, dispositivos iOS (iPhone e $\mathrm{iPad}$ ), dispositivos Windows Phone e computadores pessoais. Dessa forma, foram excluídos os tweets que possivelmente tenham sido gerados e publicados por "robôs" ou bot - um perfil automatizado que não é controlado diretamente por uma pessoa.

Foram extraídas as informações que compõem as variáveis de estudo, subdivididas em dois grupos. No $1^{\text {o }}$ grupo, as características da postagem foram: data e horário da postagem; fonte/dispositivo utilizado; contagem de caracteres do tweet; contagem de retweets (compartilhamentos); contagem de curtidas/favoritos do post; idioma do post; unidade da federação (UF) do post; conteúdo de cada tweet. No $2^{2}$ grupo identificou-se as características do perfil do usuário: nome do usuário/autor da postagem; número de seguidores do usuário; total de curtidas do usuário; contabilização de contas verificadas/figuras públicas.
Foram analisados os perfis de contas mais influentes (maior número de seguidores e perfil verificado) que publicaram postagens relacionadas aos termos de estudo, além das postagens mais populares que correspondiam aos termos de pesquisa durante esse período. Em seguida, foi elaborada nuvem de palavras com as hashtags mais comuns e as palavras-chave mais frequentes (número de ocorrências) nas postagens.

Em todo o processo de mineração de dados foi utilizado as funções da linguagem de programação estatística R, do pacote rtweet elaborado por Kearney $\mathrm{MW}^{(10)}$. O processo compreende as seguintes etapas: elaboração da estratégia de busca e coleta; coleta dos dados; pré-processamento da coleta; montagem de um banco de dados com as variáveis supracitadas; formulação de tabelas, gráficos e ilustrações com indicadores para as análises.

Cabe destacar que, para o desenvolvimento desta pesquisa, a mineração de dados também compreende uma mineração de texto de uma expressiva quantidade de informações. Para tanto, foi elaborado um corpus - uma coleção de textos, com base no conteúdo das postagens (tweets) para obtenção de uma análise textual quantitativa, contagem de palavras e nuvem de palavras por frequência de palavras - os 100 termos mais recorrentes. Para isso, utilizou-se o software livre Interface de $R$ pour les Analyses Multidimensionnelles de Textes et de Questionnaires (IRAMUTEQ) ${ }^{(11)}$.

O estudo utilizou exclusivamente dados (tweets) recuperados de mídia social de domínio público. Portanto, nenhuma aprovação ética foi necessária e está isento da necessidade de parecer de Comitê de Ética em Pesquisa. Ademais, a pesquisa está em conformidade com os termos de serviços e as políticas de desenvolvedor da API do Twitter.

\section{Resultados}

$\mathrm{Na}$ Tabela 1 são mostradas as características e a distribuição das postagens de usuários do Twitter. Nela, é possível observar que a maioria das publicações foram feitas utilizando dispositivos de celular (1.932 - 74\% - Android e iOS). 
Não obstante ser uma campanha virtual nacional, nota-se a publicação de postagens em outros idiomas, como espanhol e inglês. O idioma classificado como indeterminado $(929$ - 35,6\%) refere-se às postagens que utilizam exclusivamente hashtags (\#) e publicações de imagens e/ou vídeos acompanhados das hashtags; isso faz com que a programação identifique o idioma como indeterminado, entretanto, a grande maioria destes estão em português.

Tabela 1 - Caracterização das postagens no Twitter referentes às hashtags. Brasil. 5-13 maio 2020. $(\mathrm{N}=2613)$

\begin{tabular}{|c|c|c|c|c|c|}
\hline \multicolumn{6}{|c|}{ Variáveis } \\
\hline Dispositivos & $\mathbf{n}$ & $\%$ & Status da conta & $\mathbf{n}$ & $\%$ \\
\hline Android & 1337 & 51,2 & Não verificada & 2530 & 96,8 \\
\hline Desktop/Web & 567 & 21,7 & Verificada & 83 & 3,2 \\
\hline iOS & 595 & 22,8 & & & \\
\hline \multirow[t]{3}{*}{ Outros } & \multirow[t]{3}{*}{114} & \multirow[t]{3}{*}{4,4} & & & \\
\hline & & & Localização** & $\mathbf{n}$ & $\%$ \\
\hline & & & \multirow{2}{*}{$\begin{array}{l}\text { Região Centro-Oeste } \\
\text { Região Nordeste }\end{array}$} & 191 & 15,9 \\
\hline Idioma & $\mathbf{n}$ & $\%$ & & 244 & 9,3 \\
\hline Espanhol & 15 & 0,6 & Região Norte & 59 & 2,3 \\
\hline Inglês & 9 & 0,3 & Região Sudeste & 587 & 22,5 \\
\hline Português & 1660 & 63,5 & Região Sul & 117 & 4,5 \\
\hline Indeterminada* & 929 & 35,6 & Não informada & 1415 & 54,2 \\
\hline \multicolumn{6}{|c|}{ Estatísticas descritiva } \\
\hline Média & & Mínimo & Máximo & \multicolumn{2}{|c|}{ Soma } \\
\hline 127,7 & \multicolumn{2}{|r|}{21} & 282 & \multicolumn{2}{|c|}{333.732} \\
\hline 5,75 & \multicolumn{2}{|r|}{-} & 2.216 & \multicolumn{2}{|c|}{15.020} \\
\hline 1,17 & \multicolumn{2}{|r|}{-} & 245 & \multicolumn{2}{|c|}{3.050} \\
\hline $2.529,5$ & \multicolumn{2}{|r|}{-} & 1.279 .856 & \multicolumn{2}{|c|}{6.609 .606} \\
\hline
\end{tabular}

Fonte: Elaboração própria.

Nota: Sinal convencional utilizado:

- Dado numérico igual a zero não resultante de arredondamento.

* Refere-se às postagens que utilizam exclusivamente hashtags (\#) ou imagens e/ou vídeos acompanhados das hashtags: \#LuteComoUmaEnfermeira, \#EnfermagemEuValorizo.

* Localização de origem do post, agrupada de acordo com a divisão regional em macrorregiões (cinco grandes regiões) do Brasil.

Quanto à análise descritiva, o número mínimo de caracteres dos tweets foi 21 , o que coincide com os caracteres da hashtag: \#enfermagemeuvalorizo. Houve mais de 18 mil interações (somadas as curtidas e os compartilhamentos dos tweets), sendo que os autores das postagens atingem um público (audiência) de quase 7 milhões de perfis - estimada pelo somatório do número de seguidores dos autores das postagens (Tabela 1).

As contas são predominantemente não verificadas, o que significa maior participação de pessoas físicas/comuns, isto é, que não são figuras públicas e/ou contas de páginas oficiais de órgãos e instituições. Dos perfis que informaram sua localização no Twitter, 22,5\% localizam-se nos estados da região Sudeste do país, seguida da região Nordeste $(9,3 \%)$, conforme evidenciado na Tabela 1 e ilustrado no mapa coroplético da Figura 1.

A Figura 1 ilustra a distribuição geográfica da participação nacional na campanha e a nuvem de palavras mostra o grau de frequência dos 100 vocábulos mais utilizados nas postagens em todo o país durante o período analisado. Nesse sentido, quanto mais a palavra foi utilizada, maior (mais evidente) é a palavra no gráfico digital. Além das hashtags monitoradas na pesquisa e que apareceram com maior frequência,destacam-se: \#HUdaUSPemLuta, \#AposentadoriaEspecial, \#PisoSalarial, \#SOSTrabalhadoresDaSaúde. 
Figura 1 - Mapa coroplético da distribuição geográfica do número de postagens, conforme Unidade da Federação (a) e nuvem de palavras dos termos mais frequentes dessas postagens (b). Brasil - 2020

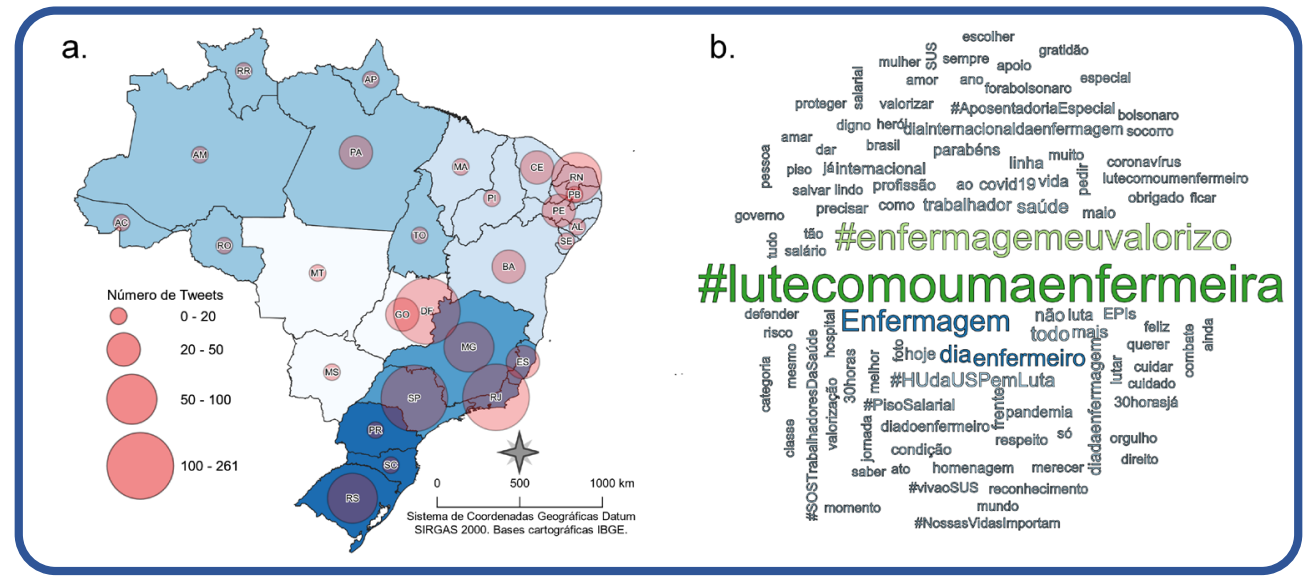

Fonte: Elaboração própria.

A campanha virtual agendada para o dia 12 de maio de 2020 registrou algumas poucas publicações dias antes, com pico no dia da campanha e decremento acentuado no dia seguinte, 13 de maio de 2020 - dia da coleta dos dados. Quando observada a distribuição temporal pela variável hora, tem-se o pico dos posts às 16 horas, com 604 publicações (Figura 2).

Figura 2 - Distribuição temporal das postagens no Twitter, segundo a data (a) e hora (b). Brasil - 2020

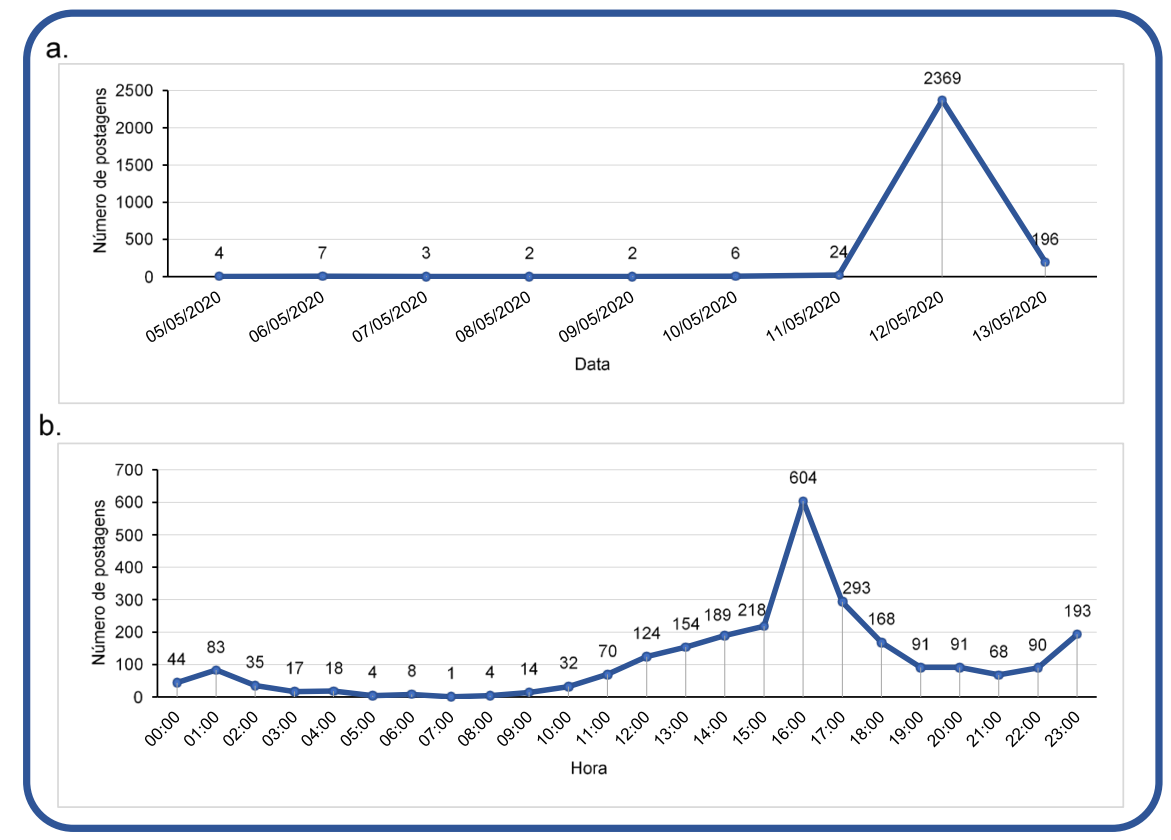

Fonte: Elaboração própria.

Para demonstrar os principais temas e assuntos que se relacionaram com as hashtags da campanha, foram apresentadas e ordenadas as principais publicações, segundo a influência (aferida pelo número de seguidores) do autor da postagem. O principal influenciador apresentou mais de 1 milhão de seguidores no Twitter e sua publicação teve 643 curtidas e 170 compartilhamentos - trata-se de uma figura pública do universo da música (Pop rock). Dos demais influenciadores, seis são ligados à área da política, um youtuber (Drag Queen), uma atriz brasileira 
e o outro, trata-se de um perfil oficial do Comitê Internacional da Cruz Vermelha. Os conteúdos das postagens versam sobre vários temas com destaque para: regulamentação da jornada de trabalho; equipamentos de proteção individual; combate à COVID-19; defesa da força de trabalho majoritariamente feminina da Enfermagem e luta por direitos (Quadro 1).

Quadro 1 - Ordenamento dos dez principais influenciadores, segundo número de seguidores. (continua)

\begin{tabular}{|c|c|c|c|}
\hline Ordem & Influenciador & Conteúdo de texto da postagem & Engajamento \\
\hline 1 & Nando Reis $\checkmark$ & $\begin{array}{l}\text { •Regulamentação da jornada de trabalho (que } \\
\text { aguarda votação na Câmara há } 20 \text { anos) } \\
\text {-Instituição de piso salarial } \\
\text { •EPI's para exercer sua profissão com segurança } \\
\text { Vamos lutar pela valorização dos } \\
\text { enfermeiros, técnicos e auxiliares. } \\
\text { \#LuteComoUmaEnfermeira } \\
\text { \#EnfermagemEuValorizo }\end{array}$ & $\begin{array}{l}\text { Seguidores: } 1.279 .856 \\
\text { Curtidas: } 643 \\
\text { Compartilhamentos: } 170\end{array}$ \\
\hline 2 & $\begin{array}{l}\text { David Miranda } \\
\checkmark\end{array}$ & $\begin{array}{l}\text { Amanhã, } 12 \text { de maio, é Dia Internacional } \\
\text { da Enfermagem. Como uma das iniciativas } \\
\text { para homenagear e defender essas e esses } \\
\text { profissionais que estão na linha de frente } \\
\text { no combate à covid-19, faremos um tuitaço } \\
\text { às 20h30. \#LuteComoUmaEnfermeira } \\
\text { \#SOStrabalhadoresdasaúde \#ForaBolsonaro }\end{array}$ & $\begin{array}{l}\text { Seguidores: } 330.757 \\
\text { Curtidas: } 475 \\
\text { Compartilhamentos: } 64\end{array}$ \\
\hline 3 & $\begin{array}{l}\text { Sâmia Bomfim } \\
\checkmark\end{array}$ & $\begin{array}{l}\text { Que o Dia Internacional da Enfermagem } \\
\text { seja um marco de mudança para os mais de } \\
2,2 \text { milhões profissionais brasileiros que até } \\
\text { hoje não possuem piso salarial e jornada de } \\
\text { trabalho regulamentada. É urgente garantir } \\
\text { direitos e condições dignas de trabalho. } \\
\text { \#LuteComoUmaEnfermeira }\end{array}$ & $\begin{array}{l}\text { Seguidores: } 288.016 \\
\text { Curtidas: } 324 \\
\text { Compartilhamentos: } 24\end{array}$ \\
\hline 4 & $\begin{array}{l}\text { Luciana Genro } \\
\checkmark\end{array}$ & $\begin{array}{l}\text { Saúde é palavra feminina! As mulheres } \\
\text { são maioria entre os trabalhadores } \\
\text { da saúde, estão na linha de frente } \\
\text { do combate ao coronavírus nas mais } \\
\text { diversas profissões e precisam do nosso } \\
\text { apoio. \#SOSTrabalhadoresDaSaúde } \\
\text { \#LuteComoUmaEnfermeira } \\
\text { \#NossasVidasImportam \#ImpeachmentJá } \\
\text { Neste Dia Internacional da Enfermagem } \\
\text { mais uma vez lembramos estes profissionais } \\
\text { que são verdadeiros heróis e heroínas } \\
\text { do combate à pandemia. Assim como } \\
\text { todas e todos os trabalhadores da } \\
\text { saúde. \#SOSTrabalhadoresDaSaúde } \\
\text { \#LuteComoUmaEnfermeira } \\
\text { \#NossasVidasImportam \#ImpeachmentJá }\end{array}$ & $\begin{array}{l}\text { Seguidores: } 265.490 \\
\text { Curtidas: } 87 \\
\text { Compartilhamentos: } 7\end{array}$ \\
\hline 5 & Lorelay Fox $\checkmark$ & \#LuteComoUmaEnfermeira & $\begin{array}{l}\text { Seguidores: } 213.127 \\
\text { Curtidas: } 2.216 \\
\text { Compartilhamentos: } 245\end{array}$ \\
\hline 6 & $\begin{array}{l}\text { Luiza Erundina } \\
\checkmark\end{array}$ & $\begin{array}{l}\text { Por trás das máscaras, há famílias. } \\
\text { \#LuteComoUmaEnfermeira } \\
\text { \#AuxilioParaSaudeJa }\end{array}$ & $\begin{array}{l}\text { Seguidores: } 185.082 \\
\text { Curtidas: } 48 \\
\text { Compartilhamentos: } 4\end{array}$ \\
\hline
\end{tabular}


Quadro 1 - Ordenamento dos dez principais influenciadores, segundo número de seguidores. (conclusão)

\begin{tabular}{|c|c|c|c|}
\hline Ordem & Influenciador & Conteúdo de texto da postagem & Engajamento \\
\hline 7 & Erika Kokay $\checkmark$ & $\begin{array}{l}\text { Nossa gratidão às/aos profissionais de } \\
\text { enfermagem que estão travando uma luta } \\
\text { histórica contra o vírus e em defesa da vida. } \\
\text { \#LuteComoUmaEnfermeira }\end{array}$ & $\begin{array}{l}\text { Seguidores: } 152.939 \\
\text { Curtidas: } 229 \\
\text { Compartilhamentos: } 57\end{array}$ \\
\hline 8 & $\begin{array}{l}\text { Comitê } \\
\text { Internacional da } \\
\text { Cruz Vermelha } \\
\checkmark\end{array}$ & $\begin{array}{l}\text { Neste\#DiaDoEnfermeiro, vamos agradecer } \\
\text { quem trabalha na linha de frente salvando nossas } \\
\text { vidas. } \\
\text { ser protegidos. \#enfermagemeuvalorizo } \\
\text { Qnfermeiros e enfermeiras devem } \\
\text { Quem salva vidas deve ser protegido. Não } \\
\text { atacado. } \\
\text { \#enfermagemeuvalorizo \#DiaDoEnfermeiro }\end{array}$ & $\begin{array}{l}\text { Seguidores: } 132.948 \\
\text { Curtidas: } 90 \\
\text { Compartilhamentos: } 27\end{array}$ \\
\hline 9 & Patricia Pillar $\checkmark$ & \#lutecomoumaenfermeira \#auxilioprasaudeja & $\begin{array}{l}\text { Seguidores: } 128.266 \\
\text { Curtidas: } 80 \\
\text { Compartilhamentos: } 3\end{array}$ \\
\hline 10 & Jaques Wagner & $\begin{array}{l}\text { Sou casado com uma enfermeira e tenho em } \\
\text { Fátima um exemplo diário da nobreza deste } \\
\text { ofício, caracterizado pela dedicação e pelo } \\
\text { cuidado com o próximo. } \\
\text { \#DiadaEnfermagem \#12demaio } \\
\text { \#LuteComoUmaEnfermeira } \\
\text { \#EnfermagemEuValorizo } \\
\text { \#SenadorDeTodosNós \#JaquesWagner }\end{array}$ & $\begin{array}{l}\text { Seguidores: } 94.616 \\
\text { Curtidas: } 18 \\
\text { Compartilhamentos: } 2\end{array}$ \\
\hline
\end{tabular}

Fonte: Elaboração própria.

Conta Verificada/Figura pública.

No Quadro 2, as postagens foram ordenadas pela quantidade de interações acerca do post, independente do número de seguidores, isto é, maior número de curtidas. Um dos influenciadores registrou mais de 2 mil curtidas em um post. Dos influenciadores listados, seis são figuras públicas - têm contas verificadas $(\checkmark)$-, e quatro são perfis de pessoas comuns. O conteúdo das postagens apresenta, entre outros assuntos: as vítimas do coronavírus e os profissionais de Enfermagem mortos; a valorização e a reivindicação de direitos.

Quadro 2 - Ordenamento dos dez principais influenciadores, segundo número de curtidas.

(continua)

\begin{tabular}{|c|l|l|l|}
\hline Ordem & \multicolumn{1}{|c|}{ Influenciador } & \multicolumn{1}{|c|}{ Conteúdo de texto da postagem } & \multicolumn{1}{|c|}{ Engajamento } \\
\hline 1 & Lorelay Fox $\checkmark$ & Ver Quadro 1 & $\begin{array}{l}\text { Curtidas: } 2.216 \\
\text { Compartilhamentos: } 245 \\
\text { Seguidores: 213.127 }\end{array}$ \\
\hline 2 & Nando Reis $\checkmark$ & Ver Quadro 1 & $\begin{array}{l}\text { Curtidas: } 643 \\
\text { Compartilhamentos: } 170 \\
\text { Seguidores: } 1.279 .856\end{array}$ \\
\hline 3 & $\begin{array}{l}\text { David Miranda } \\
\checkmark\end{array}$ & Ver Quadro 1 & $\begin{array}{l}\text { Curtidas: } 475 \\
\text { Compartilhamentos: } 64 \\
\text { Seguidores: 330.757 }\end{array}$ \\
\hline 4 & $\begin{array}{l}\text { Sâmia Bomfim } \\
\checkmark\end{array}$ & Ver Quadro 1 & $\begin{array}{l}\text { Curtidas: 324 } \\
\text { Compartilhamentos: } 24 \\
\text { Seguidores: } 288.016\end{array}$ \\
\hline
\end{tabular}


Quadro 2 - Ordenamento dos dez principais influenciadores, segundo número de curtidas. (conclusão)

\begin{tabular}{|c|c|c|c|}
\hline Ordem & Influenciador & Conteúdo de texto da postagem & Engajamento \\
\hline 5 & line & $\begin{array}{l}\text { Escolher a saúde como profissão é escolher } \\
\text { a solidariedade, a entrega e o amor ao } \\
\text { próximo. } \\
\text { Muito Grata por estar caminhando para ser } \\
\text { uma grande profissional! } \\
\text { \#LuteComoUmaEnfermeira } \\
\text { \#diadaenfermagem }\end{array}$ & $\begin{array}{l}\text { Curtidas: } 288 \\
\text { Compartilhamentos: } 29 \\
\text { Seguidores: } 3203\end{array}$ \\
\hline 6 & Jussara & $\begin{array}{l}\text { Quase } 900 \text { vidas brasileiras perdidas } \\
\text { para a \#coronavirus e junto com as } \\
\text { vidas profissionais de enfermagem que } \\
\text { estão na linha de frente. \#12demaio dia } \\
\text { Internacional do Enfermeiro estamos de } \\
\text { luto pelo Brasil. } \\
\text { Homenagem aos profissionais de } \\
\text { Enfermagem mortos. } \\
\text { \#LuteComoUmaEnfermeira }\end{array}$ & $\begin{array}{l}\text { Curtidas: } 282 \\
\text { Compartilhamentos: } 88 \\
\text { Seguidores: } 10\end{array}$ \\
\hline 7 & Cida Falabella & $\begin{array}{l}\text { Imenso orgulho e gratidão pela } \\
\text { determinação dessas trabalhadoras } \\
\text { que arriscam suas vidas para defender } \\
\text { a nossa. Não merecem só palmas, } \\
\text { mas valorização e direitos. A luta } \\
\text { da enfermeira Vanda é a nossa } \\
\text { luta! Vidas indígenas importam! } \\
\text { \#LuteComoUmaEnfermeira }\end{array}$ & $\begin{array}{l}\text { Curtidas: } 231 \\
\text { Compartilhamentos: } 46 \\
\text { Seguidores: } 18.659\end{array}$ \\
\hline 8 & Erika Kokay $\checkmark$ & Ver Quadro 1 & $\begin{array}{l}\text { Curtidas: } 229 \\
\text { Compartilhamentos: } 57 \\
\text { Seguidores: } 152.939\end{array}$ \\
\hline 9 & $\begin{array}{l}\text { Benedita da Silva } \\
\checkmark\end{array}$ & $\begin{array}{l}\text { DIRETO DE BRASÍLIA! Com } \\
\text { boneco gigante, manifestantes fazem } \\
\text { ATO EM SOLIDARIEDADE às famílias } \\
\text { dos } 12 \text { mil mortos em decorrência } \\
\text { da covid-19, no Brasil, CONTRA } \\
\text { Bolsonaro e pedem mais investimento } \\
\text { na saúde. \#LuteComoUmaEnfermeira } \\
\text { \#diainternacionaldaenfermagem } \\
\text { \#EnfermagemEuValorizo }\end{array}$ & $\begin{array}{l}\text { Curtidas: } 228 \\
\text { Compartilhamentos: } 64 \\
\text { Seguidores: } 74.950\end{array}$ \\
\hline 10 & ana banana & $\begin{array}{l}12 \text { de maio - Dia Internacional da } \\
\text { Enfermagem } \\
\text { Sorry, mas eu vou enaltecer essa área } \\
\text { incrível e extremamente necessária da } \\
\text { equipe de saúde } \\
\text { \#LuteComoUmaEnfermeira } \\
\text { \#EnfermagemEuValorizo }\end{array}$ & $\begin{array}{l}\text { Curtidas: } 221 \\
\text { Compartilhamentos: } 45 \\
\text { Seguidores: } 163\end{array}$ \\
\hline
\end{tabular}

Fonte: Elaboração própria

Conta Verificada/Figura pública.

\section{Discussão}

O monitoramento de mídias sociais tem se tornado cada vez mais importante para diversas áreas do conhecimento e a mensuração do engajamento on-line tem impacto para análises críticas e comprovações científicas no campo dos estudos digitais em saúde ${ }^{(12-14)}$.

Sendo assim, faz-se necessário que a Enfermagem conceba a realidade dos engajamentos 
nas plataformas sociais como fenômeno da pós-modernidade que influencia a disseminação de informações que podem, por suposto, refletir nas representações sociais construídas e mantidas sobre as causas da profissão ${ }^{(15)}$.

No presente estudo, foi observado predomínio de postagens utilizando dispositivos de celular, com maior participação de usuários localizados na região Sudeste do país. Desse modo, a utilização de dispositivo móvel conectado à Internet acelera a dinâmica de informação em grupos que se amplificam por meio das hahstags em seus hiperlinks, e agregam pessoas com interesses de conteúdo comum ${ }^{(15-16)}$.

Essas novas formas de interações poderão, como evidenciam os resultados, ser utilizadas para amplificar a comunicação e favorecer interações entre as pessoas/perfis das mídias sociais. Com efeito, pode-se atingir alcances globais ou de forma capilarizada por diversos contextos de um país com dimensões continentais, tal qual o Brasil.

A utilização dessas plataformas é permeada também por interesses que movimentam inclusive questões relacionadas à política e lideranças em esferas internacional e nacional. Nesse sentido, nas últimas eleições, em diferentes contextos, foram amplamente utilizadas como ferramentas para impulsionar ou desqualificar trajetórias políticas mediante a disseminação de informações e notícias falsas ${ }^{(15)}$.

No contexto e nos achados deste estudo, dentre os termos relacionados às hashtags em análise, destacam-se as publicações com reinvindicações de direitos trabalhistas voltados às demandas dos profissionais de Enfermagem. Além disso, faz-se necessário contextualizar a Enfermagem em uma perspectiva das sociologias das profissões que envolvem questões históricas, culturais e sociais que se ramificam nas relações de gênero, de políticas e de poder.

Desse modo, faz-se necessário compreender as nuances que envolvem as relações de poder, as lutas de classe e gênero, a fim de proporcionar o engajamento das enfermeiras na luta por direitos e, por conseguinte, alcançar o reconhecimento da profissão, a mudança na legislação, a melhoria da qualidade de vida da trabalhadora e a transformação cultural para sua valorização ${ }^{(17)}$.

O Conselho Internacional de Enfermagem (ICN) vem se mostrando preocupado com o número de profissionais de Enfermagem infectados e que morrem por COVID-19. No dia 3 de junho de 2020, a organização publicou um comunicado no qual apontava que, de acordo com os dados coletados, mais de 230.000 enfermeiros e profissionais de saúde já haviam contraído a doença e mais de 600 enfermeiros morreram pelo vírus ${ }^{(18)}$.

Segundo o COFEN e o ICN, o Brasil é o país com o maior número de mortes por COVID-19 entre enfermeiros e profissionais de saúde no mundo. Em um levantamento realizado até o dia 26 de maio de 2020, o Brasil havia registrado 157 óbitos de profissionais de Enfermagem, ficando à frente dos Estados Unidos, que registrou 146 óbitos e do Reino Unido com 77 óbitos $^{(6,18)}$. Entretanto, esse número vem aumentando no Brasil, e em 2 de novembro de 2020, o Observatório da Enfermagem, que é uma plataforma criada pelo COFEN para monitorar e divulgar os casos suspeitos ou confirmados de profissionais infectados pela COVID-19, registrava 41.926 casos reportados e 454 mortes.

O número elevado de mortes entre profissionais de Enfermagem em relação a outros países ressalta a importância da discussão sobre a valorização dos profissionais de saúde tendo em vista as condições de trabalho da categoria no Brasil. Isso porque, desde o início da pandemia, os Conselhos de Enfermagem receberam mais de 7.000 denúncias sobre a falta de EPI e a sobrecarga de trabalho associada ao subdimensionamento profissional ${ }^{(19)}$.

Sendo assim, é importante ressaltar que para reduzir os impactos dessa doença, de modo a garantir a quantidade e a qualidade adequada de profissionais da saúde no combate à COVID19, torna-se necessário antepor cuidados relacionados às prevenções de doenças e promoção da saúde desses trabalhadores nesse contexto ${ }^{(20)}$.

Vale destacar que as trabalhadoras enfermeiras, técnicas e auxiliares de enfermagem são em sua maioria mulheres. Portanto, sofrem 
desvalorização devido ao machismo enraizado nas relações de trabalho e de poder $^{(17)}$. Outrossim, as implicações sexistas no âmbito laboral e das profissões mantêm a ideologia de que o trabalho executado pela mulher tem menor valor e qualificação do que o trabalho executado pelo homem.

Nessa direção, o presente estudo mostrou que dos assuntos publicados por usuários do Twitter, os mais recorrentes foram exatamente a regulamentação da jornada de trabalho, os EPIs, o combate da COVID-19, a defesa da força de trabalho feminina e a luta por direitos. Temas esses que evidenciam parte dos impactos da pandemia para esses trabalhadores. Em paralelo ao exposto, no Brasil, a categoria profissional vem pleiteando, por décadas, medidas relacionadas ao piso salarial, redução da jornada de trabalho para 30 horas semanais e valorização da profissão, a fim de proporcionar condições adequadas para maior qualidade da assistência em diversos cenários de atuação da Enfermagem evidenciando prevenção, promoção, recuperação e tratamento de problemas de saúde no âmbito individual e coletivo das sociedades ${ }^{(21)}$.

Nesse sentido, de algum modo, pôde-se perceber que essas revindicações ganharam maior visibilidade nesse momento histórico que, apesar de ter em seu cerne todo o processo de caos humanitário mundial disparado pela pandemia, desencadearam um ato/campanha virtual em prol da Enfermagem, que alcançou significativa repercussão no Twitter. Isso foi observado tendo em vista o que os achados apontaram sobre a participação de influenciadores, dentre eles, figuras públicas, com milhares de seguidores no Twitter, cujos posts tiveram diversas interações (curtidas e compartilhamentos) entre os usuários da plataforma.

Do exposto, depreende-se o entendimento de que a desvalorização da Enfermagem implica na própria fragilidade dos sistemas de saúde e finca-se na perpetuação de injustiças sociais culturalmente reforçadas nos cenários trabalhistas ocupados pela categoria ${ }^{(21)}$. Portanto, faz-se necessário compreender que o contexto atual da pandemia fez emergir, com maior alcance social, questões que já estavam latentes no dia a dia dos profissionais da Enfermagem em todo o país.

Além disso, chama atenção para as novas formas de mobilização social, possibilitadas pelo uso extensivo de tecnologias de informação no cotidiano dos brasileiros, trazendo à tona novas possibilidades de dinamizar o engajamento da população em lutas de semelhante grau de importância cujas visibilidades também são fundamentais para a construção de uma sociedade mais justa.

Dentre as limitações do estudo, destaca-se que a escolha de uma única mídia social para monitoramento da campanha virtual pode subestimar o alcance real da campanha, de perfil de influenciadores e de sua relevância geral. Entretanto, a opção pelo Twitter deve-se ao fato de sua interface ser amplamente aberta na cessão de $\operatorname{dados}^{(9)}$.

A recenticidade do método de pesquisa e a falta de pesquisas anteriores sobre o tema abordado, com o emprego de tecnologias e ferramentas utilizadas no presente estudo, também pode ser apontada como uma das limitações. Sobre este último aspecto, uma das explicações para essa lacuna de estudos, deve-se ao fato de que as pesquisas que empregam esse tipo de análise estão mais presentes na área da Comunicação Digital, Big Data e Métodos Digitais ${ }^{(7,9)}$.

Assim, trata-se de uma oportunidade para a ciência da Enfermagem, a busca por novas investigações com base nesse prisma. Por fim, o ordenamento dos dez principais influenciadores e seus respectivos tweets para apresentar o engajamento na campanha foi arbitrativo, determinado, a priori, pela ausência de uma definição estrita da literatura, embora tenha resultado em boa variedade de dados em todas as regiões do país.

\section{Conclusão}

A realização da campanha virtual de valorização da Enfermagem, impulsionada pelas hashtags e publicações analisadas, obtiveram importante, numericamente e relevante visibilidade para os usuários do Twitter, no território nacional. Além disso, apresentou grande diversidade de 
temáticas e reinvidicações para a profissão, evidenciadas pelos termos e frequência de palavras que apresentaram amplo alcance da mídia social na Internet.

Ainda assim, é preciso considerar a longevidade e a durabilidade do interesse acerca desses temas e dos pleitos da campanha. As mídias sociais e os ambientes virtuais contam histórias. Para quebrar paradigmas e mudar a realidade da Enfermagem é necessário muito mais que visibilidade. Fazer e transformar a história depende, entre outros fatores, de articulações específicas dos atores envolvidos nessa construção.

Em suma, conhecer o processo de participação ativa nas publicações midiáticas no Twitter sobre a campanha de valorização da Enfermagem trouxe à luz diversas batalhas travadas especialmente no campo da prestação de cuidados em saúde, tendo em vista, principalmente, o contexto de adoecimento e o alto índice de óbitos desses profissionais pela COVID-19. Nesse sentido, novos estudos podem contribuir para uma compreensão mais aprofundada da repercussão de cada uma dessas lutas nas mídias sociais e o quanto se pode depreender dos impactos pelo uso dessas tecnologias para a disseminação de informações na atualidade.

Destarte, esse movimento de considerável repercussão, disparado pelo então contexto mundial de atravessamento dessa pandemia, sinaliza para a necessidade de se pensar medidas que possam garantir o fôlego dessas pautas, de modo a conquistar lugar na agenda política nacional. Defende-se que dar visibilidade à profissão em campanhas virtuais, como a que foi aqui retratada, é um grande passo no que tange à sensibilização da sociedade e a possibilidade de (des)construção de certas representações sociais. Entretanto, precisa-se avançar na concretização de medidas efetivas, para que as condições de trabalho da categoria de Enfermagem sejam essencialmente modificadas, refletindo de fato na valorização da profissão no Brasil.

\section{Colaborações:}

1 - concepção, projeto, análise e interpretação dos dados: Tiago Oliveira de Souza,
Hércules Rigoni Bossato, Isabela Barboza da Silva Tavares Amaral e Fernanda Teles Morais do Nascimento;

2 - redação do artigo e revisão crítica relevante do conteúdo intelectual: Tiago Oliveira de Souza, Hércules Rigoni Bossato, Isabela Barboza da Silva Tavares Amaral, Fernanda Teles Morais do Nascimento, Ítalo Rodolfo Silva e Luana dos Santos Costa;

3 - aprovação final da versão a ser publicada: Tiago Oliveira de Souza, Isabela Barboza da Silva Tavares Amaral e Fernanda Teles Morais do Nascimento.

\section{Referências}

1. Zhu N, Zhang D, Wang W, Xingwang Li, Yang B, Song $\mathrm{J}$, et al. A novel Coronavirus from patients with pneumonia in China, 2019. N Engl J Med. 2020;382(8):727-33. DOI: 10.1056/NEJMoa2001017

2. World Health Organization. Naming the coronavirus disease (COVID-19) and the virus that causes it [Internet]. Geneva (CH); 2020 [cited 2020 Apr 20]. Available from: https://www.who. int/emergencies/diseases/novel-coronavirus-2019/ technical-guidance/naming-the-coronavirusdisease-(covid-2019)-and-the-virus-that-causes-it

3. Brasil. Ministério da Saúde. Plano de contingência nacional para a infecção humana pelo novo Coronavírus COVID-19 [Internet]. Brasília (DF); 2020 [cited 2020 Jun 1]. Available from: https:// www.unasus.gov.br/especial/covid19/pdf/19

4. Miranda FMA, Santana LL, Pizzolato AC, Saquis LMM. Working conditions and the impact on the health of the nursing professionals in the context of covid-19. Cogitare enferm. 2020;25:e72702. DOI: http://dx.doi.org/10.5380/ ce.v25i0.72702

5. Souza NVDO, Gonçalves FGA, Pires AS, David HMSL. Neoliberalist influences on nursing hospital work process and organization. Rev Bras Enferm. 2017;70(5):912-9. DOI: http://dx.doi. org/10.1590/0034-7167-2016-0092

6. Conselho Federal de Enfermagem. Brasil é o país com mais mortes de enfermeiros por Covid-19 no mundo [Internet]. Brasília (DF); 2020 [cited 2020 Jun 1]. Available from: http://www.cofen.gov.br/ brasil-e-o-pais-com-mais-mortes-de-enfermeirospor-covid-19-no-mundo-dizem-entidades_80181. html 
7. Omena JJ, editor. Métodos Digitais: teoria-prática-crítica [Internet]. Lisboa: Instituto de Comunicação da Nova; 2020 [cited 2020 May 1]. Available from: https://www.icnova.fcsh.unl.pt/ en/icnova-metodos-digitais/

8. Organização Mundial de Saúde. Descritores em Ciências da Saúde (DeCS) [Internet]. Genebra $(\mathrm{CH}) ; 2020$ [cited 2020 Apr 23]. Available from: http://decs.bvsalud.org

9. Silva T, Stabile M, organizadores. Monitoramento e pesquisa em mídias sociais: metodologias, aplicações e inovações [Internet]. São Paulo: Uva Limão; 2016 [cited 2020 Apr 20]. Available from: https://www.ibpad.com.br/o-que-fazemos/ publicacoes/monitoramento-e-pesquisa-emmidias-sociais-metodologias-aplicacoes-einovacoes/

10. Kearney MW. rtweet: Collecting and analyzing Twitter data. R package version 0.7.0. J Open Source Softw. 2019;4(42):1829. DOI: 10.21105/ joss.01829

11. Camargo B, Justo A. IRAMUTEQ: Interface de $\mathrm{R}$ pour les Analyses Multidimensionnelles de Textes et de Questionnaires [Computer software]. Version 0.7. Boston (US); 2014 [cited 2020 Apr 20]. Available from: http://www.iramuteq.org

12. Rufai SR, Bunce C. World leaders' usage of Twitter in response to the COVID-19 pandemic: a content analysis. J Public Health (Oxf). 2020;42(3):510-6. DOI: https://doi.org/10.1093/ pubmed/fdaa049

13. Velasco E. Disease detection, epidemiology and outbreak response: the digital future of public health practice. Life Sci Soc Policy. 2018;14(1):7. DOI: $10.1186 /$ s40504-018-0071-4

14. Vicari S, Iannelli L, Zurovac E. Political hashtag publics and counter-visuality: a case study of \#fertilityday in Italy. Inf Commun Soc. 2018;23(9):1235-54. DOI: 10.1080/1369118X. 2018.1555271
15. Paula LT, Silva TRS, Blanco YA. Pós-verdade e Fontes de Informação: um estudo sobre fake news. Rev Conhecimento em Ação [Internet]. 2018 [cited 2020 Jun 10];2(1):93-110. Available from: https:// revistas.ufrj.br/index.php/rca/article/view/16764

16. Vieira AC, Harisson DM, Bueno M, Guimarães N. Use of the Facebook ${ }^{\text {TM }}$ social network in data collection and dissemination of evidence. Esc Anna Nery. 2018;22(3):e20170376. DOI: https:// doi.org/10.1590/2177-9465-ean-2017-0376

17. Dias MO, Souza NVDO, Penna LHG, Gallasch $\mathrm{CH}$. Perception of nursing leadership on the fight against the precariousness of working conditions. Rev esc enferm USP. 2019;53:e03492. DOI: http://dx.doi.org/10.1590/ s1980-220x2018025503492

18. International Council of Nurse. More than 600 nurses die from COVID-19 worldwide. Press Information [Internet]. Geneva (CH); 2020 [cited 2020 Jun 11]. Available from: https://www.icn.ch/ news/more-600-nurses-die-covid-19-worldwide

19. Conselho Federal de Enfermagem. Brasil responde por $30 \%$ das mortes de profissionais de Enfermagem por covid-19 [Internet]. Brasília (DF); 2020 [cited 2020 Jun 17]. Available from: http://www.cofen.gov.br/brasil-responde-por-30das-mortes-de-profissionais-de-enfermagem-porcovid-19_80622.html

20. Rodrigues NH, Silva LGA. Gestão da pandemia Coronavírus em um hospital: relato de experiência profissional. J nurs health. 2020;10(esp):e20104004. DOI: https://doi.org/10.15210/jonah.v10i4.18530

21. World Health Organization. State of the world's nursing 2020: investing in education, jobs and leadership [Internet]. Geneva (CH); 2020 [cited 2020 Jun 1]. Available from: https://www.who.int/ publications-detail/nursing-report-2020

Recebido: 15 de setembro de 2020

Aprovado: 8 de novembro de 2020

Publicado: 29 de janeiro de 2021

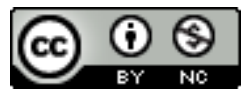

A Revista Baiana de Enfermagem utiliza a Licença Creative Commons - Atribuição-NãoComercial 4.0 Internacional. https://creativecommons.org/licenses/by-nc/4.0/ Este artigo é de acesso aberto distribuído sob os termos da Licença Creative Commons (CC BY-NC). Esta licença permite que outros remixem, adaptem e criem a partir do seu trabalho para fins não comerciais. Embora os novos trabalhos tenham de lhe atribuir o devido crédito e não possam ser usados para fins comerciais, os usuários não têm de licenciar esses trabalhos derivados sob os mesmos termos. 